\title{
The Kondo effect in bosonic spin liquids
}

\author{
Serge Florens, Lars Fritz, and Matthias Vojta \\ Institut für Theorie der Kondensierten Materie, Universität Karlsruhe, 76128 Karlsruhe, Germany
}

(Dated: July 17, 2018)

\begin{abstract}
In a metal, a magnetic impurity is fully screened by the conduction electrons at low temperature. In contrast, impurity moments coupled to spin-1 bulk bosons, such as triplet excitations in paramagnets, are only partially screened, even at the bulk quantum critical point. We argue that this difference is not due to the quantum statistics of the host particles but instead related to the structure of the impurity-host coupling, by demonstrating that frustrated magnets with bosonic spinon excitations can display a bosonic version of the Kondo effect. However, the Bose statistics of the bulk implies distinct behavior, such as a weak-coupling impurity quantum phase transition, and perfect screening for a range of impurity spin values. We discuss implications of our results for the compound $\mathrm{Cs}_{2} \mathrm{CuCl}_{4}$, as well as possible extensions to multicomponent bosonic gases.
\end{abstract}

Magnetic impurities can serve as powerful probes of the elementary excitations of the host system in which they are implanted. The well-studied situation of a spin$1 / 2$ impurity antiferromagnetically coupled to electronic quasiparticles leads to the Kondo effect, a screening of the magnetic moment at low temperature [1]. Subsequently, embedding of impurities in strongly interacting quantum systems, like high-temperature superconductors, quantum antiferromagnets, or pseudogap metals has attracted a lot of experimental and theoretical work [2-8].

Models of impurity moments interacting with collective modes of quantum magnets turn out to be particularly interesting. Consider a spin system, such as the Heisenberg bilayer [9], that can be tuned through a continuous quantum phase transition (QPT) between a Néel-ordered and a paramagnetic ground state. Close to the transition, critical fluctuations can be modelled by a standard $\mathrm{O}(3) \phi^{4}$ theory. The quantum-disordered phase has gapped spin-1 bosonic excitations, $a_{\mathbf{k} \alpha}(\alpha=x, y, z)$, which represent the dynamics of the bulk order parameter, $\phi_{\alpha} \propto\left(a_{\alpha}+a_{\alpha}^{\dagger}\right)$. The magnetic impurity $\vec{S}$ is coupled to the order parameter at the impurity site through $\mathcal{H}_{\text {imp }}=\gamma \vec{S} \cdot \vec{\phi}(\mathbf{0})$. As the $\phi$ fluctuations are gapped, the low-energy properties of the impurity are only weakly influenced by the bulk, and the impurity susceptibility displays Curie behavior, $T \chi_{\mathrm{imp}}(T)=S(S+1)$. Surprisingly, such behavior, albeit with a renormalized Curie constant, survives at the host quantum critical point (QCP) where the host gap vanishes: an impurity coupled to a bath of gapless (critical) spin- 1 bosons is not completely screened [6]. This is in stark contrast to the fermionic Kondo effect, described by the Hamiltonian $\mathcal{H}_{\text {imp }}=J \vec{S} \cdot \sum_{\sigma \sigma^{\prime}} c_{\sigma}^{\dagger}(\mathbf{0}) \vec{\tau}_{\sigma \sigma^{\prime}} c_{\sigma^{\prime}}(\mathbf{0}) / 2$, where $\vec{\tau}$ are the Pauli matrices and $c_{\sigma}^{\dagger}(\mathbf{0})$ creates a conduction electron with spin $\sigma=\uparrow, \downarrow$ at the impurity site. Physically, the two situations are different because the bosonic field $\vec{\phi}(\mathbf{0})$ acts as a (classical) fluctuating magnetic field which is unable to fully screen the moment, whereas the fermions can strongly bind to the impurity to form a quantum singlet.

The purpose of this paper is to show that this fundamental difference in the impurity behavior is not due to the quantum statistics of the bulk particles, but instead related to the structure of the impurity-host vertex. We shall demonstrate that bosonic host systems, such as frustrated critical magnets with a spin-liquid ground state and elementary spinon excitations, can quench an extra moment at low temperature, showing a true Kondo effect [17]. We introduce a new class of quantum impurity models, with superficial similarity to the fermionic Kondo model, but with bosonic spin-1/2 bulk particles. Remarkably, the bosonic nature of the host implies now markedly different properties at both weak and strong coupling, as we prove using scaling analysis, solvable toy models and a full large- $N$ solution. An experimental motivation lays also in the insulating spin system $\mathrm{Cs}_{2} \mathrm{CuCl}_{4}$, in which broad neutron scattering spectra have been attributed to the presence of spin- $1 / 2$ excitations $[10,11]$.

Frustrated magnets. For certain classes of frustrated magnets with half-integer spin per unit cell, a $\phi^{4}$ theory fails to describe the critical properties near the order-disorder transition. In the following, we will focus on a special kind of spin liquid, in which lowenergy excitations are deconfined spin- $1 / 2$ bosons, which were introduced in relation with two classes of models: anisotropic triangular magnets with incommensurate correlations [11-13] (which model the compound $\mathrm{Cs}_{2} \mathrm{CuCl}_{4}$ ), and frustrated magnets with spin- $1 / 2$ per unit cell [14]. In both cases, spinons are liberated at the QCP separating the paramagnetic from the Néel-ordered phase (although the case considered in [12] shows deconfined spinons in the whole paramagnetic phase). The effective low-energy theory for the bulk QPT takes the form

$$
\mathcal{S}_{\text {bulk }}=\frac{1}{g} \int_{0}^{\beta} \mathrm{d} \tau \int \mathrm{d}^{d} \mathbf{x} \sum_{\sigma}\left[\left|\partial_{\tau} z_{\sigma}\right|^{2}+\left|\nabla_{\mathbf{x}} z_{\sigma}\right|^{2}\right]
$$

where $z_{\sigma}$ are spinon (or $\mathrm{CP}^{1}$ ) fields with linear dispersion and constrained by $\sum_{\sigma}\left|z_{\sigma}\right|^{2}=1$. Here, gauge fluctuations are assumed to be gapped and are neglected in (1), which is only vindicated [12] for the QPT between incommensurate order and spin liquid in the anisotropic triangular lattice (gapless non-compact gauge fluctuations are important in the critical behavior proposed 
in [14]). The above constraint implies nevertheless strong interactions among the bulk bosons. Importantly, the $z_{\sigma}$ are fractional objects in terms of the physical order parameter: $\vec{\phi}=\sum_{\sigma \sigma^{\prime}} z_{\sigma}^{\dagger} \vec{\tau}_{\sigma \sigma^{\prime}} z_{\sigma^{\prime}} / 2$. This implies a large bulk anomalous dimension $\eta$ for the order parameter, i.e., the spin susceptibility at criticality follows $\chi^{-1}(\omega, \mathbf{k}) \propto\left(\omega^{2}-\mathbf{k}^{2}\right)^{1-\eta / 2}$, with $\eta=d-1$ at Gaussian level (note that the standard $\mathrm{O}(3) \phi^{4}$ theory has a tiny anomalous dimension in $d=2$ ). With the bulk theory (1) the simplest $\mathrm{SU}(2)$-invariant impurity action reads:

$$
\mathcal{S}_{J}=\int_{0}^{\beta} \mathrm{d} \tau J \vec{S} \cdot \sum_{\sigma \sigma^{\prime}} z_{\sigma}^{\dagger}(\mathbf{0}) \frac{\vec{\tau}_{\sigma \sigma^{\prime}}}{2} z_{\sigma^{\prime}}(\mathbf{0})+\mathcal{S}_{\text {Berry }}[\vec{S}] .
$$

Note that certain strong-coupling effects, i.e. high-energy bulk excitations created locally by the impurity (like nontrivial vortex fluctuations), are neglected.

Weak-coupling analysis. Bare perturbation theory in $J$, neglecting bulk interactions, yields logarithmic divergencies in $d=2$. This suggests a weak-coupling renormalization group (RG) treatment. Importantly and in contrast to the fermionic Kondo problem, a potential scattering term, $\mathcal{S}_{V}=\int d \tau(V / 4) \sum_{\sigma} z_{\sigma}^{\dagger}(\mathbf{0}) z_{\sigma}(\mathbf{0})$, is generated in the process of renormalization. At two-loop order, we find the following field-theoretic beta functions:

$$
\begin{aligned}
& \beta(j) \equiv \frac{d j}{d \log \mu}=\epsilon j-v j-\frac{1}{2} j^{3}+O\left(j^{5}\right), \\
& \beta(v) \equiv \frac{d v}{d \log \mu}=\epsilon v-\frac{1}{2} v^{2}-\frac{3}{2} j^{2}+O\left(j^{4}\right),
\end{aligned}
$$

where $J=(2 / g) \mu^{-\epsilon} j, V=(2 / g) \mu^{-\epsilon} v, \mu$ is the renormalization scale, and $\epsilon=d-2$ (for non-interacting bulk bosons). Notice that $\beta(j)$ does not contain even powers of $j$ [18], as the action is invariant under $J \rightarrow-J$ and $z_{\sigma} \rightarrow \epsilon_{\sigma \sigma^{\prime}} z_{\sigma^{\prime}}^{\dagger}$; furthermore the $j^{3}$ term is relevant for bosons. The solution of (3) is depicted in Fig. 1: For $\epsilon>0$ it shows a QCP between a local-moment (LM) phase, $J=v=0$, and a presumed strong-coupling phase (to be analyzed below); the RG flow is somewhat similar to the fermionic pseudogap Kondo model $[7,8]$ with density of states $\rho(\omega) \propto|\omega|^{\epsilon}$. For $\epsilon<0$ a transition is observed only in the presence of non-zero bare $v$ (which stabilizes a bound state, denoted BS), otherwise run-away flow to strong coupling obtains. Finally for $\epsilon=0$, the QCP disappears, leaving only flow towards strong coupling, not unlike the fermionic Kondo problem in metals. These results are strikingly different from the behavior of impurities coupled to gapless spin-1 bosons [6], where the coupling $\gamma$ flows to a stable intermediate-coupling fixed point, rendering a phase transition and true Kondo screening absent.

Incorporating bulk interactions modifies the scaling dimensions of $j$ and $v$, as these are determined by the local bulk spin correlations, $\chi(\tau) \propto 1 / \tau^{d-1+\eta}$; this gives $\epsilon=(d-3+\eta) / 2$ in Eqs. (3). Notice that the QCP in the
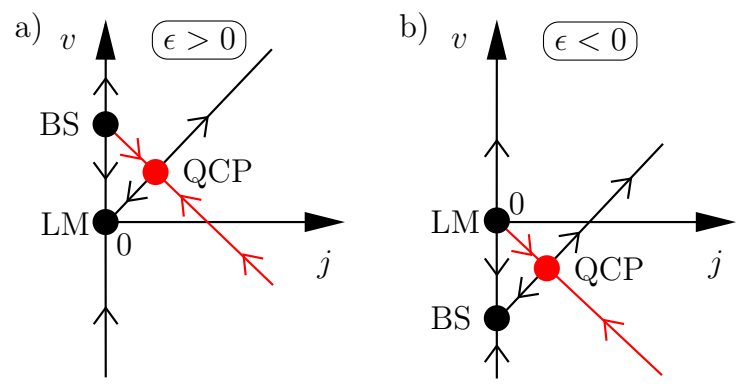

FIG. 1: (color online) Impurity RG flow of the bosonic Kondo model in dimension $d=2+\epsilon$ (for free bulk bosons). For $\epsilon>0$, the critical line crosses the $v=0$ axis, and a quantum phase transition is possible. In the presence of bulk interactions, the bulk anomalous dimension dictates the weak-coupling behavior, so that in $d=2$ figure a) [b)] applies to $\eta>1[\eta<1]$.

incommensurate magnet has $\eta>1$ in $d=2[11,12]$, i.e. a boundary quantum phase transition will occur, Fig. 1a.

Unfortunately, a consistent RG including the bulk constraint $\sum_{\sigma}\left|z_{\sigma}\right|^{2}=1$ cannot be performed (in contrast to the theory of Ref. 6). Interestingly, two transformations allow to map our original model onto a theory where both bulk and boundary interactions can be perturbatively controlled: (i) relax the hard constraint on the $z_{\sigma}$ in favor of a repulsive $u\left|z_{\sigma}\right|^{4}$ term for the spinons; (ii) perform a reversed Schrieffer-Wolff transformation as done in Ref. 8 for the pseudogap Kondo model. This leads to an effective bosonic Anderson model, where both $u$ and the hybridization term are marginal in $d=3$ which allows for an epsilon expansion; details will appear elsewhere.

Strong-coupling analysis. Let us turn to the strongcoupling limit, and ask whether screening can really occur at low temperature. Unfortunately, even when reducing the problem to a single site, our bosonic Kondo model remains complicated. However, we can introduce solvable toy models that illustrate the general strong-coupling aspects. In the first example, we replace the $z_{\sigma}$ spinons by canonical bosons $a_{\sigma}$, described by the Hamiltonian $H_{1}=U\left(\sum_{\sigma} a_{\sigma}^{\dagger} a_{\sigma}\right)^{2}+J \vec{S} \cdot \sum_{\sigma \sigma^{\prime}} a_{\sigma}^{\dagger} \vec{\tau}_{\sigma \sigma^{\prime}} a_{\sigma^{\prime}} / 2$. This is trivially diagonalized, and shows two crucial new aspects of the bosonic case which lack in the fermionic Kondo model: (i) without bulk interaction $(U=0)$, the model is ill-defined in the sense that no Pauli principle prevents the "collapse" of an infinite number of bosons onto the impurity; (ii) when $U>J / 2$, screening is possible for a range of impurity spin values $S<S_{c} \equiv J /(2 U-J)$, and underscreening obtains otherwise. This is due to the fact that spin- $1 / 2$ bosons can recombine into larger spin objects, whose size is bounded due to the local $U$ term.

Next we verify that these results also apply to the initial model based on the $z_{\sigma}$ spinons, as they are not canonical bosons [cf. the dynamic term in Eq. (1)]. We use a second toy model, now based on the $z_{\sigma}$ bosons, where the initial $\mathrm{O}(4)$ symmetry is replaced by $\mathrm{O}(2) \times \mathrm{O}(2)$ using a simplified constraint $\left|z_{\sigma}\right|^{2}=1$. This is resolved by setting 
$z_{\sigma}=\exp \left(i \phi_{\sigma}\right)$, and the one-site bosonic Kondo model thus becomes $H_{2}=-g(\partial / \partial \theta)^{2}+(J / 2) \exp (i \theta) S^{-}+$h.c. , where $\theta \equiv \phi_{\uparrow}-\phi_{\downarrow}$, and $S^{ \pm}=S^{x} \pm i S^{y}$. $H_{2}$ is diagonalized by a unitary transformation $\widetilde{H}_{2}=U H_{2} U^{-1}$, with $U=\exp \left(i \theta S^{z}\right)$. For an impurity spin $S=1 / 2$, the ground-state wave function is found to be unique and reads $\langle\theta \mid \Psi\rangle=\exp (-i \theta / 2)|\uparrow\rangle-\exp (i \theta / 2)|\downarrow\rangle$, demonstrating that the $z_{\sigma}$ spinons can succeed in forming a zero-entropy state with a quantum spin.

Large- $N$ approach. In order to put the previous arguments in a global perspective, we now develop a nonperturbative large- $N$ approach. The present problem can be generalized to a larger symmetry, by choosing $\vec{S}$ to be in the conjugate representation of $\mathrm{SU}(N)$ (in order to allow for $\mathrm{SU}(N)$ singlet formation). Eq. (2) now reads:

$$
\mathcal{S}_{J}=\int_{0}^{\beta} \mathrm{d} \tau \sum_{\sigma} b_{\sigma}^{\dagger}\left(\partial_{\tau}-\mu\right) b_{\sigma}-\frac{J}{N} \sum_{\sigma \sigma^{\prime}} b_{\sigma}^{\dagger} b_{\sigma^{\prime}} z_{\sigma}^{\dagger}(\mathbf{0}) z_{\sigma^{\prime}}(\mathbf{0})
$$

where Schwinger bosons $b_{\sigma}(\sigma=1 \ldots N)$, satisfying the constraint $\sum_{\sigma} b_{\sigma}^{\dagger} b_{\sigma}=S N$, have been introduced ( $\mu$ is the related Lagrange multiplier). Alternatively, we could have taken a full Schwinger boson solution of the bulk plus impurity problem, which - after integrating out high-energy bulk modes - yields a similar effective theory. Now, the interaction term in Eq. (4) can be decoupled. At $N=\infty$, the problem is controlled by a static saddle point, with three mean-field parameters: the chemical potential $\mu$, a bond variable $Q \equiv\left\langle z_{\sigma}^{\dagger}(\mathbf{0}) b_{\sigma}^{\dagger}\right\rangle$ which characterizes magnetic correlations between the bulk and the impurity, and a bulk mass term $\lambda(\mathbf{x})$ conjugate to $z_{\sigma}^{\dagger}(\mathbf{x}) z_{\sigma}(\mathbf{x})$ which enforces the constraint on the $z_{\sigma}$. Notice that a space dependence of $\lambda(\mathbf{x})$ is induced by $J$ around the impurity. From (4) we obtain the effective impurity Green's function of the $b_{\sigma}$ bosons:

$$
G_{b}^{-1}\left(i \nu_{n}\right)=i \nu_{n}+\tilde{\mu}+Q^{2}\left[G_{z}\left(i \nu_{n}\right)-G_{z}(i 0)\right]
$$

$\left(\nu_{n} \equiv 2 \pi n / \beta\right)$ where the renormalized chemical potential $\tilde{\mu}<0$ and the bond parameter $Q$ are determined by:

$$
\frac{1}{\beta} \sum_{n}\left\{\begin{array}{l}
1 \\
Q G_{z}\left(i \nu_{n}\right)
\end{array}\right\} e^{i \nu_{n} 0^{+}} G_{b}\left(i \nu_{n}\right)=\left\{\begin{array}{l}
-S \\
-Q / J
\end{array}\right\}
$$

We have also defined the local bulk propagator $G_{z}\left(i \nu_{n}\right) \equiv$ $G_{z}\left(i \nu_{n}, \mathbf{0}, \mathbf{0}\right)$, where $G_{z}^{-1}\left(i \nu_{n}, \mathbf{x}, \mathbf{x}^{\prime}\right)=g^{-1}\left[\nu_{n}^{2}-\nabla_{\mathbf{x}}^{2}+\right.$ $\lambda(\mathbf{x})] \delta\left(\mathbf{x}-\mathbf{x}^{\prime}\right)$. The spatial dependence, induced by $\lambda(\mathbf{x})$, has to be determined self-consistently. Finally, the local susceptibility is given by $\chi_{\mathrm{loc}}(T)=T \sum_{n} G_{b}\left(i \nu_{n}\right)^{2}$.

The form of the propagator (5) allows to predict three distinct zero-temperature phases: (i) a local-moment phase (LM) with $Q=\tilde{\mu}=0$; (ii) an underscreened phase (US) with $Q \neq 0, \tilde{\mu}=0$; (iii) an exactly screened phase (ES) with $Q \neq 0, \tilde{\mu} \neq 0$ (this nomenclature will become clear shortly). The onset of a non-zero $Q$ upon lowering $T$ defines the Kondo temperature $T_{K}$. To determine the actual phase diagram as a function of the parameters $J$,
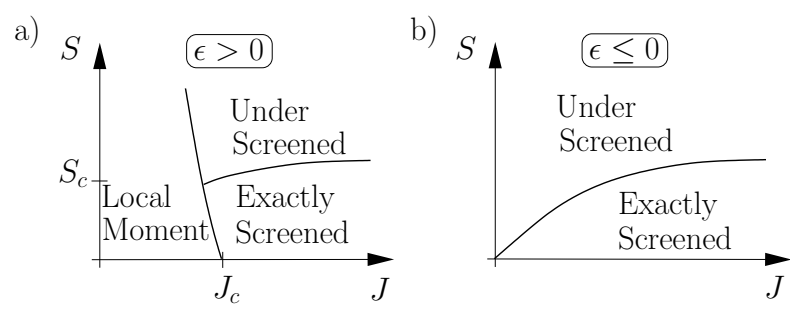

FIG. 2: Schematic phase diagrams at $N=\infty$ for the Kondo problem with bosonic spinons in dimension $d=2+\epsilon$.

$S, g$, we need to solve the full set of saddle-point equations. As a first step, the spatial dependence of $\lambda(\mathbf{x})$ will be ignored $(\lambda(\mathbf{x}) \rightarrow \lambda)$, which is justified in the regime where $Q$ is small (as discussed below). The local bulk propagator is then given by:

$$
\begin{aligned}
G_{z}\left(i \nu_{n}\right) & =\int \frac{\mathrm{d}^{d} \mathbf{k}}{(2 \pi)^{d}} \frac{g}{\nu_{n}^{2}+\lambda+k^{2}} \\
& \simeq G_{z}(i 0)-A\left|\nu_{n}^{2}+\lambda\right|^{d / 2-1}
\end{aligned}
$$

where the second identity holds at low frequency. The bulk parameter $\lambda$ is given by the usual constraint equation, see Chap. 5 of Ref. 9.

We proceed towards a general analysis of Eqs. (6) at the bulk critical point (where the gap $\sqrt{\lambda} \propto T$ vanishes as $T \rightarrow 0$ ). At high temperature, i.e. for $T>T_{K}$, we have $Q=0$. This LM phase is characterized by the Curie law $\chi_{\text {loc }}(T)=S(S+1) / T$. To determine whether this situation persists down to $T=0$, we take the $Q \rightarrow 0$ limit in Eq. (6), which shows that it does, provided $J<J_{c} \propto$ $\epsilon /(S+1 / 2)$ with $\epsilon=d-2$. The large- $N$ limit therefore confirms the existence of a critical interaction for $\epsilon>0$ in the bosonic Kondo model.

Next we turn our attention to the actual nature of the $Q \neq 0$ solutions in the regime $J>J_{c}$ and $T<T_{K}$. Using (7), $\tilde{\mu}$ is given from (6) by:

$$
-S=\int \frac{\mathrm{d} \nu}{2 \pi} \frac{e^{i \nu 0^{+}}}{i \nu+\tilde{\mu}-A Q^{2}|\nu|^{d-2}+\ldots}
$$

which converges when $\tilde{\mu} \rightarrow 0$ for $2<d<3$, and shows that there exists a critical value $S_{c}$ of $S$ above which (8) cannot be satisfied. Conversely, when $S<S_{c}, \tilde{\mu}$ saturates at $T=0$ and cuts the divergence in $\chi_{\text {loc }}$, signaling a complete screening of the local moment (in analogy to the fermionic Kondo problem). Therefore, this ES phase occurs in the bosonic model for a range of the impurity spin $S<S_{c}$, in agreement with our previous strongcoupling arguments: the bulk particles are bosonic spin$1 / 2$ spinons, which can combine such as to compensate higher spins values (up to a maximum value $S_{c}$ ). Finally, when $S>S_{c}, \tilde{\mu}$ vanishes and $\chi_{\text {loc }}$ diverges at $T=0$, signaling an undercompensation of the impurity spin. These results are summarized in the zero-temperature phase diagrams shown in Fig. 2a. All the previous analysis is well 


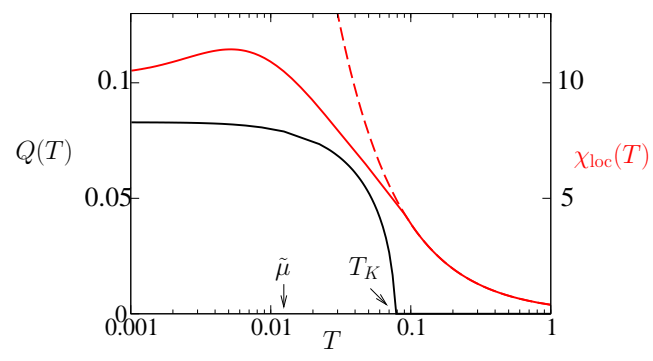

FIG. 3: (color online) Bond parameter $Q$ (lower curve) and local spin susceptibility (upper curve) as a function of temperature for $\epsilon=0.5, S=0.3<S_{c}=0.5$ and $J=2.4$. Numerical calculations were done with a momentum cutoff $\Lambda=\max (k)=10$ at the bulk critical point $g=g_{c}$. The dashed line denotes the free Curie law $\chi=S(S+1) / T$.

borne out by a full numerical solution of the saddle-point equations. The existence of an exactly screened phase is reflected in the local susceptibility which saturates at low temperature, showing a behavior strikingly similar to the fermionic Kondo effect, see Fig. 3.

So far, the large- $N$ discussion applies to space dimension $d>2$. The case $d=2$ is special. First, $J_{c}=0$, i.e. the local-moment phase is absent (the bulk anomalous dimension $\eta$ vanishes at $N=\infty$ ). Second, neglecting the spatial dependence of $\lambda(\mathbf{x})$ induced by the impurity leads to a loss of the solution of the mean field equations at low temperature, an artifact cured through the spatial variation of $\lambda(\mathbf{x})$. Although solving for the full $\lambda(\mathbf{x})$ is numerically challenging, we have established [by means of a systematic small- $Q$ expansion of (6)] that it remains short range. Indeed, the effective propagator of the bulk bosons, after integrating out the impurity, reads:

$G_{z, \text { eff }}^{-1}\left(i \nu, \mathbf{x}, \mathbf{x}^{\prime}\right)=\left(\frac{\nu^{2}-\nabla_{\mathbf{x}}^{2}+\lambda(\mathbf{x})}{g}+\frac{Q^{2} \delta(\mathbf{x})}{i \nu+\mu}\right) \delta\left(\mathbf{x}-\mathbf{x}^{\prime}\right)$

so that the impurity effect is counter-balanced by choosing $\lambda(\mathbf{x}) \simeq-\left(g Q^{2} / \mu\right) \delta(\mathbf{x})$. We have checked that this ansatz leads indeed to a stable numerical solution of the mean field equations. The resulting phase diagram is in Fig. 2b, and the susceptibility is similar to the one shown in Fig. 3.

Away from bulk criticality. Finally we comment on the impurity physics away from the bulk critical point. Moving inside the gapped paramagnet, $g>g_{c}$, shifts the critical Kondo coupling $J_{c}$ towards larger values (in particular $J_{c}$ becomes non-zero even for $\epsilon \leq 0$ ), but screening remains at low temperature, an effect which is correctly captured by our mean-field calculation. (If one is interested in the vicinity of the deconfined QCP described in Ref. 14, spinons recombine into triplet magnons above the valence bond solid phase, and our large- $N$ calculation does not apply below a typical confinement energy scale.) Inside the ordered phase, $g<g_{c}$ at low $T$, the arguments of Ref. 6 apply.

Conclusions. We have outlined a bosonic version of the Kondo effect in magnetic systems with deconfined bosonic spinons. Together with the result of Ref. 6 , we conclude that magnetic impurities are sensitive to the nature of excitations in quantum critical magnets, and may be employed as a probe of deconfined criticality [14]. Applied to $\mathrm{Cs}_{2} \mathrm{CuCl}_{4}$ we propose to study magnetic properties of dilute impurity moments (e.g. by local NMR probes) - true screening would imply the presence of bulk spinons. Finally, our results apply to the description of impurity effects [15] in multicomponent bosonic gases trapped in optical lattices [16], albeit with some important modifications due to the lack of relativistic invariance and the possible presence of a Bose condensate. Interestingly, a quadratic dispersion would imply a bare dimension $\epsilon^{\prime}=(d-2) / 2$ of the Kondo coupling, so that a non-trivial critical point could persist in $d=3$.

We thank S. Sachdev, T. Senthil and G. Zarand for valuable discussions, and K. Damle for a collaboration at the early stage of this work, particularly regarding the small- $Q$ expansion of the saddle-point equations. This research was supported by the DFG Center for Functional Nanostructures and the Virtual Quantum Phase Transitions institute in Karlsruhe.

[1] A. C. Hewson, The Kondo Problem to Heavy Fermions, Cambridge University Press, Cambridge (1996).

[2] O. P. Vajk, P. K. Mang, M. Greven, P. M. Gehring and J. W. Lynn, Science 295, 1691 (2002).

[3] D. G. Clarke, T. Giamarchi and B. I. Shraiman, Phys. Rev. B 48, 7070 (1993).

[4] J. L. Smith and Q. Si, Europhys. Lett. 45, 228 (1999).

[5] A. M. Sengupta, Phys. Rev. B 61, 4041 (2000).

[6] M. Vojta, C. Buragohain, and S. Sachdev, Phys. Rev. B 61, 15152 (2000).

[7] D. Withoff and E. Fradkin, Phys. Rev. Lett. 64, 1835 (1990).

[8] M. Vojta and L. Fritz, Phys. Rev. B 70, 094502 (2004).

[9] S. Sachdev, Quantum Phase Transitions, Cambridge University Press, Cambridge (1999).

[10] R. Coldea, D. A. Tennant, and Z. Tylczynski, Phys. Rev. B 68, 134424 (2003).

[11] S. V. Isakov, T. Senthil and Y. B. Kim, condmat/0503241.

[12] A. V. Chubukov, T. Senthil, and S. Sachdev, Phys. Rev. Lett. 72, 2089 (1994).

[13] P. Azaria, P. Lecheminant, and D. Mouhanna, Nucl. Phys. B 455, 648 (1995).

[14] T. Senthil, L. Balents, S. Sachdev, A. Vishwanath, and M. P. A. Fisher, Phys. Rev. B 70, 144407 (2004).

[15] A. Recati, P. O. Fedichev, W. Zwerger, J. von Delft and P. Zoller, Phys. Rev. Lett. 94, 040404 (2005).

[16] T. Nikuni and J. E. Williams, J. Low Temp. Phys. 133, 323 (2003).

[17] This was shown rigorously in 1D for the fermionic spinons of the Heisenberg chain [3].

[18] A relevant $j^{2}$ term in $\beta(j)$ appears for canonical $\mathrm{SU}(2)$ bulk bosons, and for $\mathrm{SU}(N)$ bulk spinons when $N>2$. 